\title{
A case of cervical esophageal perforation after transnasal gastrointestinal fibroscopy using a small-caliber fiber
}

Transnasal gastrointestinal fibroscopy using a small-caliber fiberscope (N-GIF) has been established as a safe procedure because it can prevent undesired pharyngeal reflux $[1,2]$. However, the risks and complications related to N-GIF remain unclear [1-5].

A 38-year-old man complaining of epigastralgia with a small amount of hematemesis was admitted to a private clinic and underwent emergency N-GIF (Olympus GIF-XP150N; Olympus, Tokyo, Japan), which revealed mild hemorrhagic esophagitis. During N-GIF he did not feel severe discomfort, and suffered only slight pharyngeal reflux. He had a small amount of hematemesis, dyspnea and tachycardia 20 hours after N-GIF, and hyperglycemia, disorientation, hypotension, and anuria 2 days later. CT revealed an abnormality in the left piriform sinus and mediastinal emphysema ( $\bullet$ Figs. 1, 2).

He was transferred to our center and underwent emergency conventional oral gastrointestinal endoscopy (GIF), which revealed pooling of bloody pus around the left piriform sinus with local redness and edema ( Fig. 3 ).

During the emergency operation, we found purulent inflammation around the cervical esophagus but could not find the perforation site. We diagnosed a minor perforation of the left piriform sinus and performed simple drainage. GIF performed on the 6th postoperative day revealed no ulcer ( $\bullet$ Fig. 3 ).

Most of the reported complications due to $\mathrm{N}-\mathrm{GIF}$ involve nasal bleeding [5]. There has been no report of pharyngoesophageal injury. However, because of the flexibility of the fiber, the endoscopist may not feel any resistance even when the fiber becomes wedged in the piriform sinus, and may continue to push the fiber without any resistance. Although in most cases the tip of the fiber can slip into the esophagus without any injury, it can cause a mucosal injury during such a slip. In our patient, it is thought that a nontransmural piriform injury that occurred during $\mathrm{N}$ GIF became transmural due to his clearing his throat or straining himself after N-GIF.

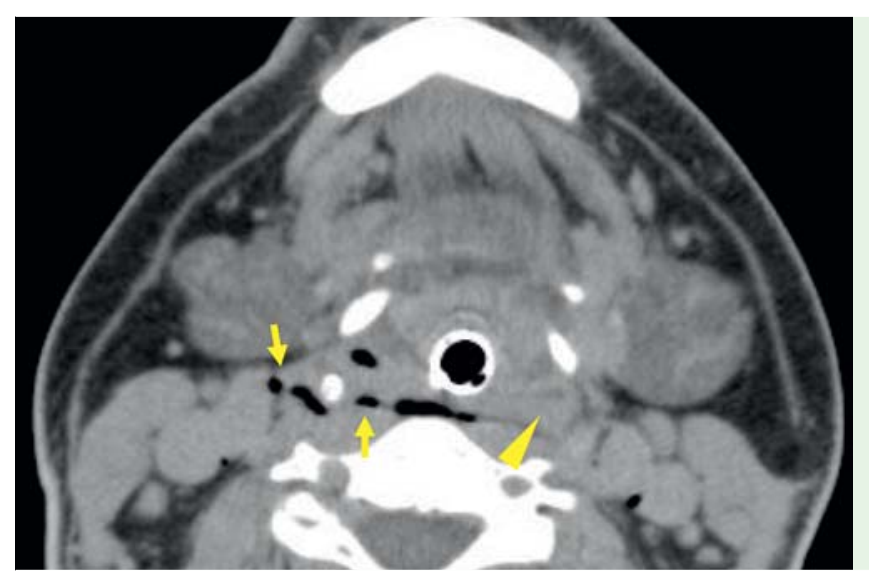

Fig. 1 Neck CT showing collapse of the left piriform sinus filled with liquid (low density material, arrowhead) and deep cervical emphysema (arrow).
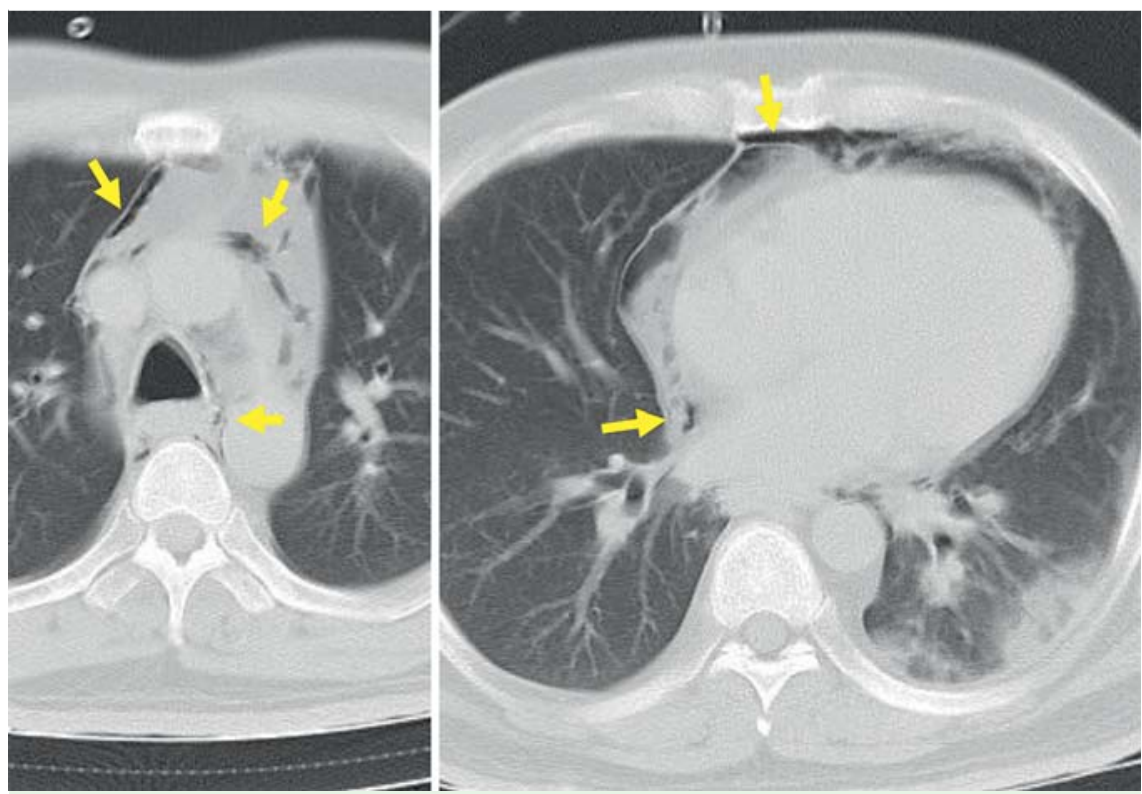

Fig. 2 Chest CT showing mediastinal emphysema spreading to the level of the heart (arrows).

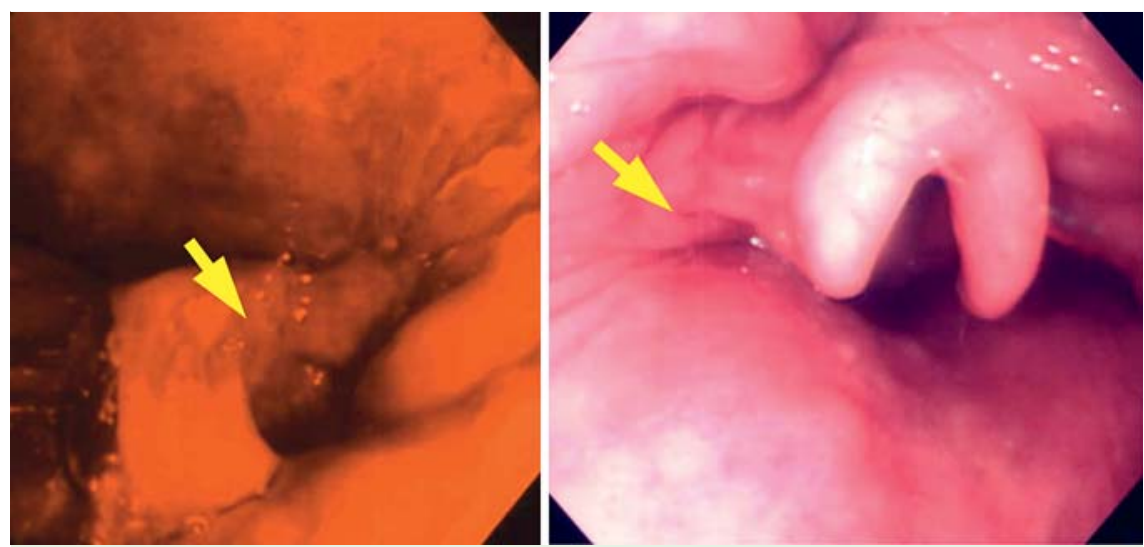

Fig. 3 Emergency conventional gastrointestinal fibroscopy with poor image on admission (left, arrow), showing pooling of bloody pus and irregular pit with severe mucosal edema around the left edge of the epiglottis; and on the 6th postoperative day (right, arrow), showing marked improvement of the lesion. 
Endoscopy_UCTN_Code_CPL_1AH_2AB

Competing interests: None

Y. Moriwaki, S. Arata, M. Iwashita, H. Toyoda, T. Kosuge, N. Suzuki Critical Care and Emergency Center, Yokohama City University Medical Center, Yokohama, Japan

\section{References}

1 Cheung J, Bailey R, Veldhuyzen van Zanten $S$ et al., CANHelp working group. Early experience with unsedated ultrathin $4.9 \mathrm{~mm}$ transnasal gastroscopy: a pilot study. Can J Gastroenterol 2008; 22: 917-922

2 Yagi J, Adachi K, Arima $N$ et al. A prospective randomized comparative study on the safety and tolerability of transnasal esophagogastroduodenoscopy. Endoscopy 2005; 37: $1226-1231$

3 Yuki M, Amano Y, Komazawa Y et al. Unsedated transnasal small-caliber esophagogastroduodenoscopy in elderly and bedridden patients. World J Gastroenterol 2009; 15: 5586- 5591

4 Tatsumi Y, Harada A, Matsumoto T et al. Current status and evaluation of transnasal. Review. Esophagogastroduodenoscopy. Dig Endosc 2009; 21: 141 - 146

5 Hagiwara S, Hirayama M, Sasaki K et al. A randomized study on the tolerability and adverse effect on cardiopulmonary parameters with esophagogastroduodenoscopy by small caliber endoscope. Gastroenterol Endosc 2008; 50: 359-368

\section{Bibliography}

DOI $10.1055 / \mathrm{s}-0030-1256211$

Endoscopy 2011; 43: E147 - E148

(c) Georg Thieme Verlag KG Stuttgart · New York . ISSN 0013-726X

\section{Corresponding author}

Y. Moriwaki, MD

Critical Care and Emergency Center Yokohama City University Medical Center 4-57 Urafune-cho, Minami-ku

Yokohama

232-0024, Japan

Fax: +81-45-2433677

qqc3@yokohama-cu.ac.jp 